UC-20d

Issued: September 1985

$L A=-10528-M S$

DE86 003297

\title{
Multipoint Thomson Scattering System and Measurements on the Los Alamos Spheromak Compact Toroid Experiment
}

\author{
Rita Gribble \\ T. R. Jarbce \\ H. W. Hoida \\ J. Lipson* \\ E.W. Newman
}

\section{DISCLAIMER}

\begin{abstract}
This report was prepared as an account of work sponsored by an agency of the United States Government. Neither the United States Government nur any agency thereof, nor any of their employees, makes any warranty, express or implied, or assumes any legal liability or responsibility for the accuracy, completeness, or usefulness of any information, apparatus, product, or process disclosed, or represents that its use would not infringe privately owned rights. Reference herein to any specific commercial product, process, or service by trade name, trademark, manufacturer, or otherwise does not necessarily constitute or imply its endorsement, recommendation, or favoring by the United States Government or any agency thereof. The views and opinions of authors expressed herein do not nesessarily state or reflect those of the United States Government or any agency thereof.
\end{abstract}

*AT\&T Bell Laboratories, 555 Union Blvd., Allentown, PA 18103.
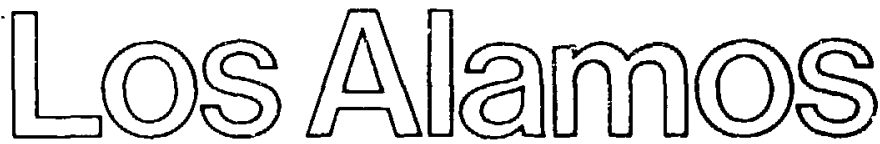

Los Alamos National Laboratory Los Alamos,New / Mexico 87545 


\title{
MULTIPOINT THOMSON SCATTERING SYSTEM AND MEASUREM YTS \\ ON THE LOS ALAMOS SPHEROMAK COMPACT TOROID EXPERIMENT
}

by

\author{
Rita Gribble, T. R. Jarboe, H. W. Hoida, \\ J. Lipson, and E. W. Newman
}

\begin{abstract}
A multipoint Thomson scattering system providing radial electron temperature and density profiles of plasma in a single shot is an on-line diagnostic on the spheromak device of CTX. The system includes a 22-joule, dual-beam ruby laser, large collection optics, and a spectrophotometer that contains a 40-mm dual chevron micro-channel-plate image intensifier tube as shutter and first stage of light amplification. A unique nethod of calibration that uses an 870-mm-long tungsten rod light source for instrument transmission and linearity measurements is described. Examples of data and temperature profiles are presented.
\end{abstract}

\section{INTRODUCTION}

A radial profile of temperature and density is important in determining the characteristics of a magnetically confined plasma in fusion experiments. A multipoint Thomson scattering system was designed and constructed to obtain these rrofiles for the CIX spheromak experiment at Los Alamos National Laboratory. The basic concept for this system was taken from work done by Bretz et al., 1 Siemon et $\mathrm{al}_{4}{ }^{2}$ and Freesis et al. ${ }^{3}$ Nevertheless, there are some significant variations such as the use of a dual-beam, 22-joule laser, a dual chevron micro-channel-plate image intensifier, and a unique calibration light source. These improvements were essential to overcome the high background light and low plasma density of the experiment.

Essential elements of the system are a ruby laser light source, collection optics, and a spectrophotometer containing a spectrograph and an electronic camera. A laser beam is used to illuminate a full radius (400 $\mathrm{mm}$ ) of the plasma. Scattered iight from the plasma radius is collected with a large reduction lens that focuses the light onto a narrow fiber-optic array. The output of the array provides an input slit for the spectrograph. 
Dispersed light from the spectiograph grating creates a two-dimensional pattern representing wavelength and spatial location. An image intensifier tube coupled to a silicon intensified target vidicon (SIT) receives this focused pattern. Output from the SIT is digitized and relayed to a computer. A radial profile of electron temperature and density for the plasma at a given time (in one shot) is then obtained.

To maximize the signal-to-noise ratio a high-power, 22-joule, dual-beam ruby laser is used. Low $f$-number, large collection, and spectrograph optics were chosen to maximize the throughput of the instrument. A spherical holographic grating is the first element in the spectrograph, to diminish undispersed stray laser light.

Currently 12 spatial locations, each with 415 wavelength channels, are being analyzed. With densities as low as $2 \times 10^{13} \mathrm{sm}^{-3}$, temperatures of 10 to about $150 \mathrm{eV}$ are measured with 75 to $90 \%$ certainty. The information provided by this device has been especially userul in determining the effects of varyins physical parameters on the experiment. It has been instrumental in finding a means for reducing impurities and increasing the electron temperature.

\section{LASER AND BEAM OPTICS}

The ruby laser is a Q-switched, high-power, low-divergence unit built by Quantel and capable of delivering 22 joules with its dual-beam output. (See Fig. 1.) After passing through the oscillator and first ruby amplifier the beam is split into two nearly equal beams. Each beam has two additional stages of amplification. The two output beams have a 0.3-mrad divergence and a diameter of $19 \mathrm{~mm}$. By using mirrors the beams are manipulated so that they become adjacent to each othe (20 mm center to center) and parallel. The beams are then focused at a plasma radius of $200 \mathrm{~mm}$ using a $2.25-\mathrm{m}$ focal length lens. The maximum width of the laser beam along the 400-mm-long scattering volume as seen by the spectrometer is $2 \mathrm{~mm}$. The maximum width in the crthogonal projection is $4 \mathrm{~mm}$. The 400-mm-long scattering volume allows a complete major-radial profile of density and temperature to be measured.

The laser beam enters the CTX vacuum tark through a high-quality optical window mounted at Brewster's angle on a sube containing bafrles to trap laser light scattered from the window. (See Fig. 2.)

A viewing dump designed to prevent reflected laser light from entering the spectrograph is mounted on the tank wall opposite the viewing port. It is fabricated of strips of heat-darkened stainless foil in a 152-mm-wide, 152-mm-deep chevron pattern.

The laser beam exits the tank into an L-shaped, 51-mm-diameter beam dump. It is fabricated of a 152-mm-wide, 152-mm-deep chevron. Entrance to the dump is through a Corning blue-glass (CS5-58) shield with a 31.75-mm aperture through which the beam passes. The first section of the $\mathrm{L}$ is $660 \mathrm{~mm}$ long and the second is $362 \mathrm{~mm}$ long. The entire length contains a series of grayed aluminum baffles. At the right-angle turn a rectangle of blue glass is mounted at a $45^{\circ}$ angle to direct the beam down the next section. At the end of this section another piece of blue glass is similarly mounted. This last piece of blue glass directs the beam upward to a mirror at $90^{\circ}$ to the glass. This mirror brings the remainder of the beam or a HeNe beam out of the syst.m for alignment purposes. 
A. Collection Optics

A $165 \mathrm{~mm}$ flat glass window provides optical access to the CTX tank. Outside the window the collection iens system is tilted by an adapter creating a viewing angle of $17^{\circ}$ above the horizontal, where the magnetic axis of the spheromak can be viewed. (See Fig. 2.) The collection lens system was designed using the ACCOS V optical design code. The system is composed of a 140-mm-diameter cemented doublet and a 170-mm field lens. The system has an effective focal length of $223.6 \mathrm{~mm}$ a $1 / \mathrm{f}$-number of 2.2 , a magnification of 0.28 , and a fleld of view of $31^{6}$. This lens 1mages the 400-mm-long scatterting volume onto the $112-\mathrm{mm}$ by $0.84-\mathrm{mm}$ fiber-optlc array. By rearranging the opposite end of the fibers a 67.2-mm by $1.4-\mathrm{mm}$ entrance slit for the spectrophotometer is formed. In the rearrangement the integrity of the location is malntained to preserve the spatial information.

\section{B. Spectrophotometer Optics}

Inside the spectrophotometer the first element is a holographic spherical-aberration-corrected diffraction grating. It has a 203-mm dlameter, a 750-mm radius of curvature, and 1650 grooves/mm. Fleld lenses before the grating were avoided in order to minimize the undispersed stray laser light in the instrument. All succeeding elements are $152 \mathrm{~mm}$ in diameter or larger. The final element is an $\mathrm{f} / 1.2,4: 1$ reduction lens, giving a final 1mage $25 \mathrm{~mm}$ on a side.

At the lmage plane of the spherical grating a mask is inserted to prevent the 6943- ruby laser light from reaching the camera where it would saturate the detector. Another mask is placed in this 1mage plane to block the intense $D \alpha$-radiation ( $6563 \AA$ ) emitted by the deuterlum plasma. The $\mathrm{D} \alpha$-mask is a neutral density $3.0 \mathrm{f} 11 \mathrm{ter}$, which reduces the intensity to an acceptable level, but allows the Da-radiation to be monftored over a full radius.

\section{Electronic Camera}

The 25-mm-square 1mage contalning the wavelength and spatial information is formed on a 40-mm-ilameter, ITT model F4150, channel-imageIntensifier-tube photocathode. The Iarge 40-mm size was chosen to maximize tine scattered 11ght throughput. The image intensifier is composed of a photocathode (S-20), dual chevron micro-channel-plates (MCP), and an output phosphor (P-20). The dual MCP 1mage intenstfier tube increases the signal-to-noise rat1o approximately an order of magnitude over a single MCP intensiffer tube. The gated image intensifier acts as a fast optical shutter ( $100 \mathrm{~ns}$ ) and provides the f1rst stage of light amplification. The phospior is coupled to the SIT faceplate with a tapered fiber-optic coupler reducer (2:1), thus f1111ng the 12.5-mm-square 1nput of the SIT.

The control chassis for the Image intensifier tube and the SIT are contained in a shielded rack located in the experimental area near the spectrophotometer housing. The control electronics for the 1mage intensifier tube include the bias- and gain-voltage power supplies and the MCP gate circult. The gate is a 100-ns, 200-volt, flat square wave pulse to forward-bias the photocathode. The SIT controller is a PARC model 1216 that supplies control voltages for the SIT and is the data communication $11 \mathrm{nk}$ between the experiment computer with its CAMAC interface and the SIT. Commands sent from the computer to the 1216 initialize the SIT scan pattern. Analog data from the SIT are digltized by the 1216 and sent to the computer. Two line driver-recelver chassis transmit information by twisted pair lines 
to and from the CTX control and data acquisition system's CAMAC interface. Communication with the CIX control room for timing functions is by fiber-optic links.

D. Spectrophotometer Housing

The spectrograph and detector system are contained in a $2-m$ by $1-m$ by $0.3-m$ aluminum light-tight housing. The optical elements including the camera are mounted on the $2-m$ by $1-m$ by $25-\mathrm{mm}$ hack plate of the housing. A triple-layer Mumetal shield protects the sensitive elec:ronics from the magnetic fields of the experiment.

E. Aligninent

All elements in the spectrograph have at least three latitudes of micrometer adjustment, making precision alignment possible. The accessibility to the large interior of the tank makes possible an easy means of ituagin the laser focus on the fiber-optic dissector. A HeNe laser whose beam path coincides with the ruby laser beam path is used to illuminate a white screen in the tank. The entrance slit of the spectrophotometer is backlit with a high-intensity lamp, and the collection optics focus the light in the tank. Adjustments are then made to the fibers such that the light is focused at the HeNe beam path. This method requires that the vacuum tank be at atmospheric pressure. Another more cumbersome method that does not require a vacuum tank opening is achieved by moving a bellows-mounted target into the HeNe laser beam path. The target is angled such that the HeNe beam makes a lorg thin line seen by the fiber array. A short-focal-length telescope is then used to view the input slit from inside the spectrophotometer. The orientation and position of the fiber array is adjusted for best aligninent and focus.

F. Timing

The gate pulse, which forward-biases the photocathode of the image intensifier, is $100 \mathrm{~ns}$ long. Fifty ns are required to fully turn on the image-intensifier photocathode. The 30-ns (FWHM) laser pulse with 10-ns fitter is centered in the last $50 \mathrm{~ns}$ of the gate pulse. Synchronization of the laser pulse and image-intensifier gate is achieved by using a fiber-optic cable of length equal to the laser path to pick of a small percentage of the laser beam and transmit it to a photodiode located near the gate circuitry. The diode's output is then compared with the gate monitor signal on a dual-beam scope. Once approximate synchronization is achieved, the gate timing is varied in small increments until maximum signal is acquired. This was accomplished using stray laser light for the signal.

G. Stabilizing Dark Current

To maintain a stable, low-level dark current and reduce noise in the SIT, a constant, relatively cool operating temperature is required. A jacket. was consiructed around the SIT to accomodate circulating chilled water to provide these conditions. An operating temperature of $9{ }^{\circ} \mathrm{C}$ for the SIT is maintained. In the normaily dry atmosphere of Los Alamos condensation as a result of the decreased temperature is seldom a problem. However, when necessary, a trickle of dry nitrogen is fed into the closed spectrophotometer to prevent condensation. 


\section{H, Spectral and Spatial Distribution}

The 12.5-mm-square raster scan of the SIT is divided into sixieen 0.78 -mm-high bands each containing five hundred $0.025-\mathrm{mm}$-wide pixels. The center 12 bands receive the data covering $400 \mathrm{~mm}$ of lasma radius. Each band views approximately $35 \mathrm{~mm}$ of the plasma. The spectrum is analyzed from $6465 \AA$ to $6915 \AA$. The longer wavelength dimension is delimited by the ruby laser mask. Each spatial location has approximately 415 usable wavelength data points. The pixel width corresponds to $1.08 \AA$, but the resolution of the instrument, as determined primarily by the entrance-slit width and the grating, is approximately $15 \AA$.

\section{IV . CALIBRATION}

\section{A. Instrument Transmission}

Relative transmission from the collection lens through the detector is determined for each wavelength and at each spatial location. An 870-mm-long, 3.2-mm-diameter tungsien rod is mounted in the tank (during calibration procedures only). It is located such that it coincides with the ruby laser beam path and is held unaer tension with a spring mount. The tank is evacuated and a voltage applied across the tungsten rod. The color cemperature of the giowing rod $(-1760 \mathrm{~K})$ is measured with a pyrometer. The tungsten wavelength emittance curve is compared with the spectrophotometer output to create a transmission factor for each pixel. The radiation from the rod is adjusted to approximately equal that observed while taking data, and the image intensifier is operated with the same gating and voltages that are used during normal data taking.

\section{B. Linearity}

The SIT is operated in a continuous scan mode that refreshes each pixel every $160 \mathrm{~ms}$. The minimum read time per pixel is $20 \mu \mathrm{s}$. The firing of the laser is set to coincide with the first track of the SIT scan pattern. A coincidence detector was designed to synchronize the firing of the machine with the SIT scan pattern ensuring corsistency in the timing of the illumination of the SIT with the location of the read beam.

For a short pulse of light the output of the SIT is a nonlinear function of the total light that is incident on the tube. ${ }^{4}$ This effect is especially important when the SIT is read only once. Because of the large number of pixels, the read time is inly slightly storter than the persistence of the SIT. Thus only one read is possible. The nonlinearity was measured over a wide range of light-signal amplitudes. (The hot tungsten rod was used as the ligin source with neutral-density filters to vary the light levela) The nonlinearity of gain vs light inpui intensity is uniform over the face of the SIT. Therefore one correction to the linearity function works well (accurate within 10\%) for the entire tube. In our case we used $I=A V 0.787$ for $V<50$, $I=1.88 \mathrm{AV}^{0.625}$ for $50<V<300$, and $I=3.84 \mathrm{AV}^{0} 5$ for $V>300$. V is the number of output counts, A is the transmission factor for each location, and $I$ is the incident signal. The values of $A$ vary by as much as a factor of 10. In practice only Vs between 5 and 3000 are encountered. One interesting effect is that although the output dynamic range is 600 , the input range is less than 60 (3till adequate for the diagnostic). It also should be noted that the response function was determined for the same level of dark current during calibration and data taking. Complicated physical means of correcting for the nonlinearity are possible wherein the SIT target is cocled to about $-35^{\circ} \mathrm{C}$ and a read pattern to erase, read, and restore the SIT are implemented. 
A simpler method was attempted ${ }^{5}$ wherein the cathode voltage is raised $\sim 1 \mathrm{~V}$ immediately before the read frame. The output is then linear with respect to input; however, we found a signifisant degradation in the signal-to-noise ratio. Using software to correct the problern is simple, accurate, and cost effective.

\section{Wavelength}

Wavelength calibration is accomplished for each spatial location by using a neon Geissler-tube light source, which has three strong radiation lines between 6600 and $7000 \AA$. With the masks removed the full viewable spectrum is $460 \AA$. A parabolic fit to these three nearly linear points for each spatial location is used to determine the wavelength of light hitting each pixel of the SIT. Checking these values with a monochrometer showed an error of less than $3 \AA$.

\section{DATA ANALYSIS}

Thomson scattered light is only one of several contributors to the out.put of the system. Other elements that must be removed from the data are the inherent SIT dark current, the background plasma light, and the stray ruby laser light. A few seconds after reading the data, a dark current level is automatically taken and stored. All analysis programs begin by subtracting dark current point by point from the recorded data. For each time in the discharge for which data is being taken, the plasma radiation is recorded without firing the laser. Also at least twice during each data-taking session a laser-only shot is taken to determine the laser stray-light illumination, which is usually very small. The net Thomson scattered signal is calculated by subtracting both the plasma radiation and the laser stray-light readings from the total signal of the data shot. The net signal data is analyzed with a chi-square fitting routine for temperature and density proriles. Figure 3 shows a data shot, a plasma-only shot, a laser-only shot, and the data shot minus the other two shots for one spatial location. Figure 4 shows the individual fits for the 12 radial positions. Figure 5 shows the temperature and density data obtained from one shot on the CTX experiment.

Because we have not absolutely calibrated the instrument, only a relative measurement of electron density is made. An absolute density profilu is calculated by comparing the full-radius averaged electron density determined by Thomson scattering with the diameter averaged density measured by $a$ HeNe infrared quadrature interferometer.

\section{SUMMARY}

The multipoint Thomson scattering device is operating on the CTX experiment providing valuable temperature and density profiles. Improvements over previous designs such as the high-power, dual-beam laser, large optics, dual MCP, and calibration techniques using the tungsten rod were required to make possible the single-shot, radial temperature measurements in the CTX experiment. 


\section{ACKNOWLEDGMENTS}

The authors would like to express their appreciation to J. D. Smith for his technical support and mechanical design of the spectrometer.

\section{REFERENCES}

1. N. Bretz, D. Dimock, V. Foote, D. Johnson, D. Long, and E. Tolnos, "Multichannel Thomson Scattering Apparatus," Applied Optics 17, i92 (1978).

2. R. E. Siemon, R. Kristal, K. B. Freese, P. R. Forman, F. C. Jahoda, G. I. Chandler, M. D. Bausman, D. A. Platts, in "LASL Controlled Thermonuclear Research Program, January-December 1976," K. S. Thomas, Comp., Los Alamos Scientific Laboratory report LA-7082-PR (March 1978), pp. 127-133.

3. K. B. Freese, R. S. Massey, Rita Gribble, J. D. Smith, "Multi-point Thomson Scattering Measurement for the ZT-40 Reversed Field Pinch," SPIE 288, Los Alamos Conference on Optics (1981).

4. H. Staerk, R. Mitzkus, and H. Meyer, "Performance of SIT Vidicons Whe॥ Exposed to Transient Light Signals," Applied Optics 20, No، 3 (1981).

5. G. Liesegang and P. Smith, "Improving Vidicon Linearity in the Pulsed Illumination Mode," Applied Optics 20, Nos 15 (1981). 


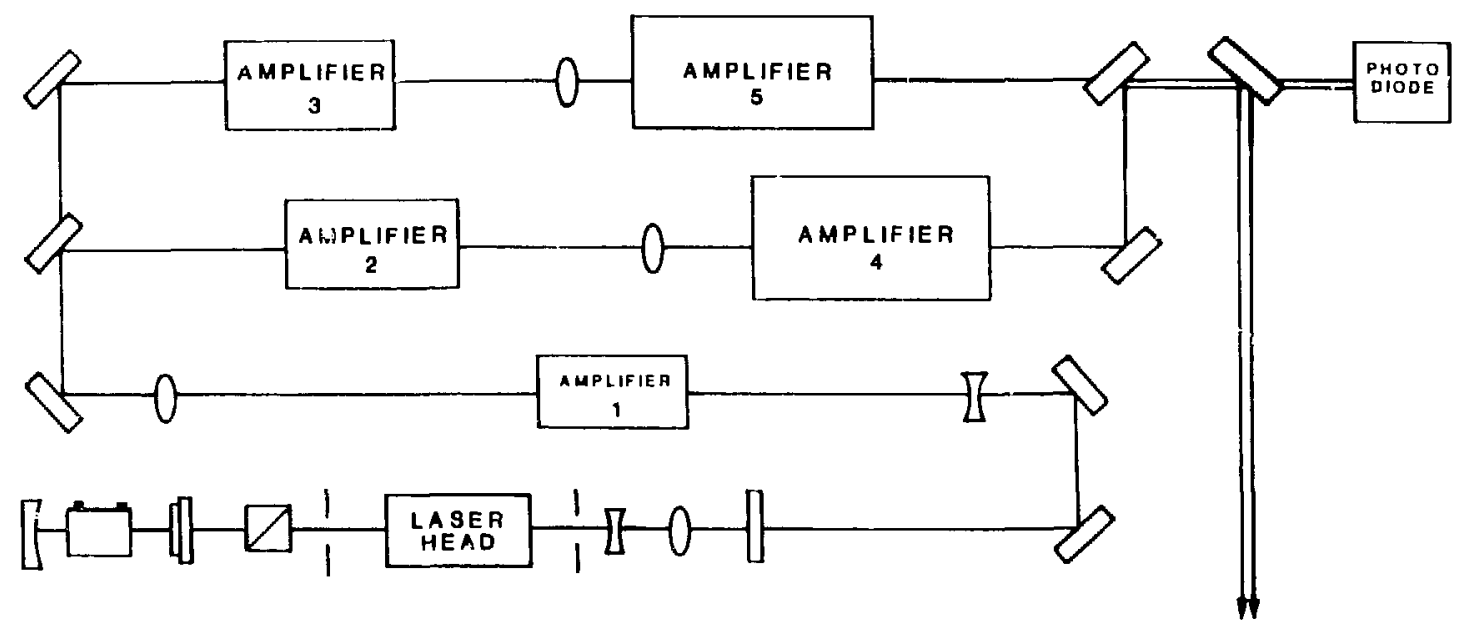

Fig. 1.

Schematic drawing of the 22-joule, dual-beam ruby laser. The oscillator consists of the rear mirror (100\%), pockel sell, quarter wave plate, Glan-Taylor polarizer, $2.3-\mathrm{mm}$ spatial filter, the laser head (6.3-mm-diameter by $135-\mathrm{mm}-1$ ing ruby rod), $2.5-\mathrm{mm}$ spatial filter, and output coupler (reflectivity 18\%). Amplifier 1 is a 9.52-mm-diameter by 152-mm ruby. Amplifiers 2 and 3 are 12.7-m-diameter by 207-mm ruby rods. Amplifiers 4 and 5 are 19-mm-diameter by 204-mm ruby rods. After amplifiers 4 and 5 the beams come to an element that is half transparent, haif mirror. The lower beam hits the mirror part and is reflected toward the $99 \%$-reflective final mirror, and the upper beam is transmitted through the other half to the final mirror. The photodiode is used as the laser power monitor. 


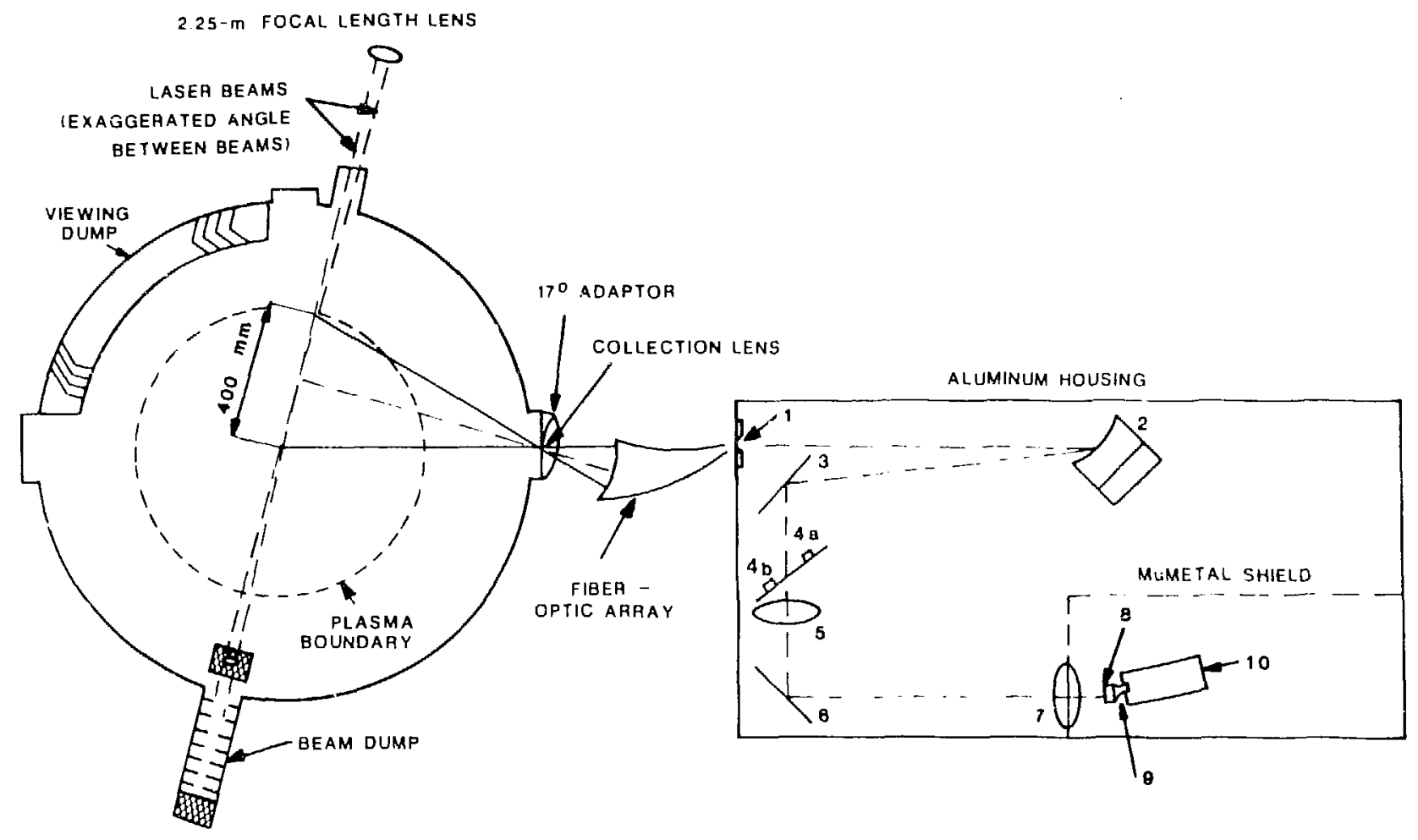

Fig.2.

Schematic drawing of the CTX multipoint Thomson scattering device: 1. input slit $(1.4 \mathrm{~mm}$ by $67.2 \mathrm{~mm})$, 2. grating, 3. mirror, 4. image plane, 4a. Damask, 4b. ruby mask located at the 6943-A wavelengti location, 5. field lens, 6. mirror, 7. reduction lens, 8 . image intensifier, 9. fiber-optic reducer coupler, and 10. SIT. 


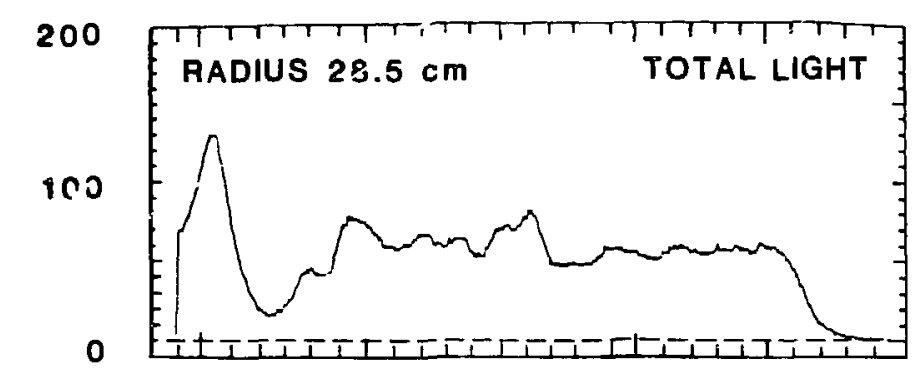

a.

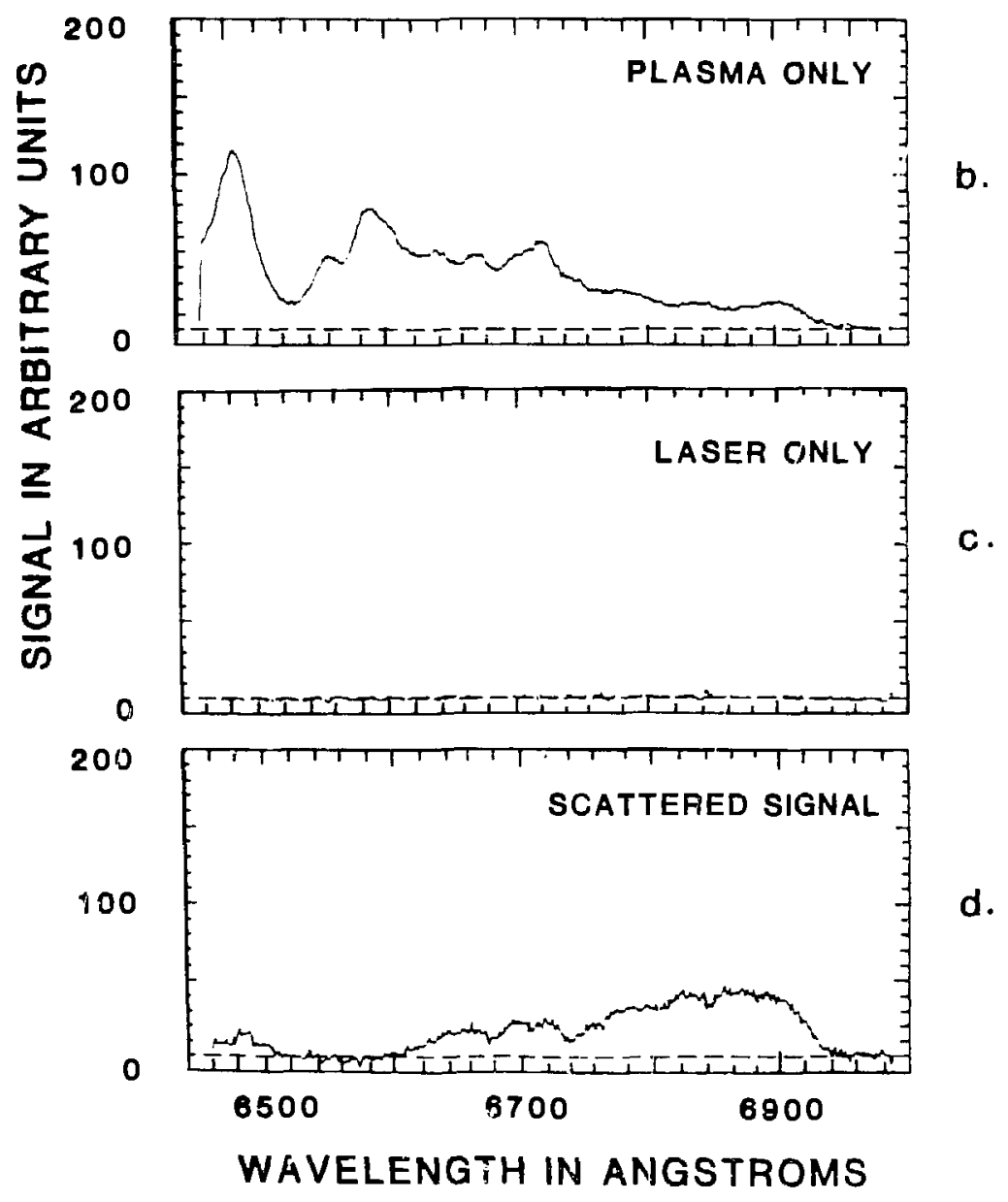

Fig. 3.

Data lised in temperature analysis: a) total light, b) plasma-only light, c) laser-only light, d) total light minus the plasma light and laser light for one spatial location (radius $=28.5 \mathrm{~cm}$ ). 


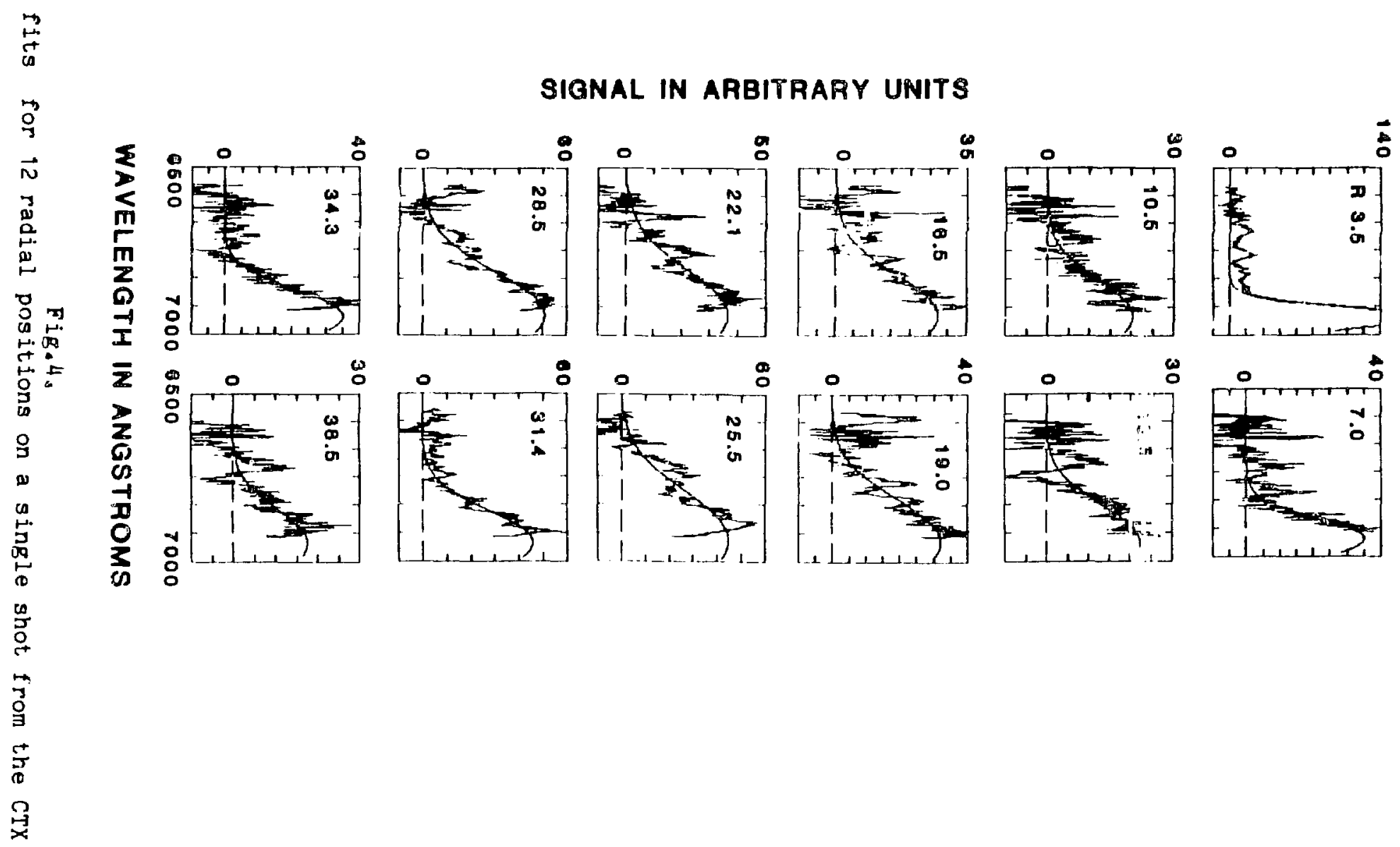




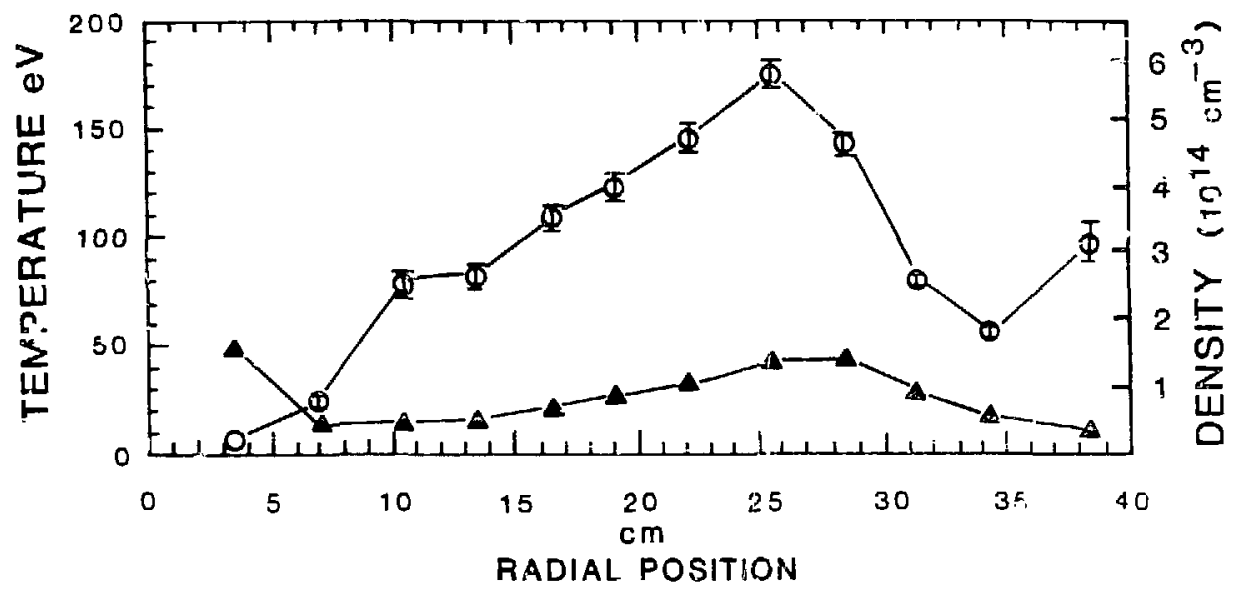

Iig. 5.

Temperature (0) and density $(\Delta)$ datid obtained from the data shown in Fig. 4. 